\title{
Laterais do Português Europeu Contemporâneo: estruturação interna, robustez de traços e dados da aquisição
}

\author{
Clara Amorim \& João Veloso \\ Universidade do Porto / CLUP
}

\begin{abstract}
This work intends to discuss the distinctive features of the laterals in Contemporary European Portuguese, in particular the features [+lateral] and [+continuous], based on language acquisition data. For this purpose, the productions of 80 typically developing children aged between 3 and 4 years and eleven months were analyzed. The results show that, after the nasals, the lateral in onset position is the first sonorant to be acquired. If the laterals are distinguished from the rhotics by the marked feature [+lateral], it would be expected that the class of the rhotics would be acquired before the laterals, since the acquisition of segments is made by the gradual acquisition of marked features and by their combination with features already acquired. The fact that $/ 1 /$ is acquired before the rhotics suggests that the feature [+lateral] is not responsible for establishing the contrast between the two classes. Based on the data analyzed, the feature [+approximant] is proposed to characterize the laterals and rhotics, distinguishing them from the other sonorants, and the feature [[ \pm continuant] to differentiate the rhotics from the laterals, the latter being characterized by the negative value of this feature.
\end{abstract}

Keywords: distinctive features, laterals, phonological acquisition, Contemporary European Portuguese.

Palavras-chave: traços distintivos, laterais, aquisição fonológica, português europeu contemporâneo.

\section{Introdução: organização e aquisição dos sistemas fonológicos}

A relevância dos traços distintivos e da sua organização hierárquica é há muito consensual na descrição fonológica das línguas (entre outros, Jakobson, Fant \& Halle 1952, Chomsky \& Halle 1968), bem como no processo de aquisição segmental. Com efeito, é principalmente no âmbito dos estudos de aquisição que a organização hierárquica dos contrastes tem sido aplicada como forma de descrever os inventários fonológicos em desenvolvimento (Ingram, 1989; Fikkert, 1994; Lazzarotto-Volcão, 2009; e.o.). No âmbito da teoria fonológica, só a partir dos finais da década de 1990 e do início da década de 2000 se assiste ao ressurgimento de estudos sobre as hierarquias de contraste, nomeadamente graças aos trabalhos de Clements $(2001,2009)$ e, paralelamente, à investigação desenvolvida na Universidade de Toronto, principalmente de B. Elan Dresher (Dresher, Piggott \& Rice 1994; Dresher 2009; e.o.).

Clements (2009) propõe que os inventários fonológicos se encontram estruturados com base nas categorias gerais de traços que surgem ao longo do processo de aquisição das línguas. Para explicar o modo como os traços se organizam nos sistemas, são propostos cinco princípios: Limitação de Traços, Economia de Traços, Evitação de Traços Marcados, Robustez e Reforço Fonológico. Centrar-nos-emos, neste trabalho, no Princípio da Robustez de Traços.

O Princípio da Robustez baseia-se na existência de uma hierarquia universal de traços. De acordo com Clements (2001, 2009), a seleção de traços na constituição dos sistemas fonológicos é feita com base nos contrastes que eles estabelecem. Os contrastes mais robustos ocorrem com mais frequência nas línguas do mundo, maximizam a saliência com menor esforço articulatório e são adquiridos antes dos traços menos robustos. 
De forma a identificar os traços mais comuns nas línguas do mundo e a organizá-los hierarquicamente, Clements $(2001,2009)$ analisa os contrastes existentes nas 451 línguas que constituíam, à data da publicação dos trabalhos citados, a base de dados UPSID da Universidade da Califórnia (UCLA Phonological Segment Inventory Database): os traços que ocupam o topo da escala são os que ocorrem nos contrastes mais frequentes, sendo, por isso, considerados robustos, enquanto os que ocupam a base são pouco robustos, já que são responsáveis pelo estabelecimento de menos contrastes. O Quadro 1 reproduz a Escala de Robustez proposta por Clements (2005/2009).

\begin{tabular}{|c|c|}
\hline \multirow[t]{4}{*}{ a. } & {$[ \pm$ soante $]$} \\
\hline & [labial] \\
\hline & [coronal] \\
\hline & [dorsal] \\
\hline \multirow[t]{2}{*}{ b. } & [ \pm contínuo $]$ \\
\hline & {$[ \pm$ posterior $]$} \\
\hline \multirow[t]{2}{*}{ c. } & [ \pm vozeado $]$ \\
\hline & {$[ \pm$ nasal $]$} \\
\hline d. & [ \pm glotal $]$ \\
\hline e. & outros \\
\hline
\end{tabular}

Quadro 1: Escala de Robustez para Traços Consonânticos (Clements 2009)

Saliente-se que, na proposta de Clements (2009), é a interação entre os cinco princípios que explica a organização dos sistemas fonológicos. Por exemplo, a interação do Princípio da Robustez com o Princípio da Economia e do Princípio da Evitação de Traços Marcados permite explicar a tendência para a organização dos sistemas sonoros com um pequeno número de traços robustos, favorecendo as combinações de traços não marcados. Desta forma, quando há traços menos robustos presentes no sistema, estes generalizam-se a outros sons, maximizando, portanto, a sua presença.

Por outro lado, embora a Escala de Robustez proposta contenha apenas traços isolados, Clements (2009: 49) refere que o grau de robustez de cada traço varia de acordo com a coocorrência com outros traços. Por exemplo, o traço [ \pm ant] é considerado pouco robusto quando coocorre com obstruintes.

No processo de aquisição da linguagem, segundo o autor, os traços mais robustos são dominados mais cedo pelas crianças. Assim, também os contrastes estabelecidos pelos traços que ocupam uma posição inferior na escala serão adquiridos só depois dos contrastes estabelecidos pelos traços que ocupam uma posição superior.

A ideia de que a construção do sistema fonológico pelas crianças em fase de aquisição da língua materna é um processo gradual que depende da frequência (e, portanto, da marcação) dos traços foi inicialmente proposta por Jakobson (1941/1968). De acordo com este autor, o sistema fonológico da criança é mais simples do que o sistema-alvo, na medida em que possui menos contrastes. Ao longo do processo de aquisição, a criança vai acrescentando contrastes, sendo os últimos adquiridos também os menos frequentes nas línguas do mundo devendo ser postulados, portanto, como os traços mais marcados (Jakobson, 1941/1968: 57).

Diversos estudos têm confirmado que a aquisição fonológica de uma língua implica o desenvolvimento gradual de representações fonológicas para os sons que a criança recebe do input e que são contrastivos na sua língua (Brown \& Matthews, 1997, e.o.). Inicialmente as representações são não especificadas, mas vão-se tornando mais detalhadas à medida que a criança domina a fonologia da língua (entre outros, Fikkert \& Levelt, 2008, Fikkert, 2010). Este processo de identificação de contrastes fonológicos e construção de representações fonológicas torna-se mais difícil quando há variação no input, dependendo mais especificamente do tipo de variação: alofónica, alomórfica ou livre (entre outros, Peperkamp \& Dupoux, 2002, Fikkert \& Freitas, 2006). 
À medida que a criança adquire contrastes fonológicos, os traços marcados tornam-se especificados, enquanto os traços não marcados continuam não especificados nas suas representações fonológicas (Clements, 2009, Altvater-Mackensen \& Fikkert, 2010). Desta forma, a análise das produções alternativas permite compreender a construção das representações fonológicas das crianças, uma vez que são motivadas pelo apagamento de um traço marcado (Fikkert 2007, Fikkert \& Levelt, 2008), e, consequentemente, testar as análises fonológicas propostas para a gramática-alvo (Chomsky, 1986).

Neste trabalho, pretendemos discutir a estruturação interna das laterais do português europeu contemporâneo à luz do Princípio da Robustez e dos dados de aquisição.

\section{As laterais no Português Europeu Contemporâneo: caracterização e aquisição}

No português europeu contemporâneo (PEC), é consensual considerar-se que a classe das soantes é composta, fonologicamente, por três consoantes nasais $(/ \mathrm{m} \mathrm{n} \mathrm{n/} /)$, os róticos $/ \mathrm{R} \mathrm{f} / \mathrm{e}$ as laterais $/ 1 \mathrm{~K} /$. Todas estas consoantes partilham o traço [+soante], que as distingue das obstruintes, mas as nasais distinguem-se das restantes soantes por serem [-contínuo, +nasal]. Já as laterais e os róticos são caracterizados pelo traço [+contínuo]. A diferença entre as duas classes assenta no traço distintivo [+lateral], atribuído às laterais (Mateus e Andrade 2000).

Do ponto de vista articulatório, a lateral coronal [-anterior] apresenta maior estabilidade, enquanto a [+anterior] é velarizada, principalmente em Coda silábica, embora vários estudos no âmbito da fonética tenham verificado que a velarização ocorre já em todas as posições silábicas (e.o., Andrade, 1998; Emiliano, 2009; Oliveira, Martins, Teixeira, Marques \& Sá-Couto, 2011). No Português do Brasil (PB), a velarização não está descrita, registando-se a glidização de /1/ em Coda.

O estatuto de [+ contínuo] atribuído às laterais pode, porém, ser discutido, dada a sua ambiguidade fonética: na articulação destas consoantes há simultaneamente uma constrição central do fluxo de ar e a sua saída livre pelas partes laterais da língua (Chomsky \& Halle, 1968; Mielke, 2005, e.o.).

Também Mateus e Andrade (2000) discutem qual o traço ([+contínuo] ou [-contínuo]) mais adequado para a caracterização das laterais do PEC. Como argumentos para a atribuição do traço [-contínuo], os autores referem o facto de, à semelhança de /n/ e /d/, a consoante /l/ ter sofrido um processo de apagamento em posição intervocálica. A caracterização das três consoantes com o traço [-contínuo] permitiria captar o seu comportamento como uma classe natural (Andrade, 1977). Por outro lado, a caracterização das laterais com o traço [- contínuo] permitiria também distinguir esta classe da classe dos róticos, que partilham com as laterais os traços [+consonântico] e [+soante]. Já a utilização do traço [+ contínuo], solução adotada por Mateus e Andrade (2000), é justificada pelo facto de a lateral /l/, tal como o rótico alveolar/s/, poder ocorrer em coda silábica, posição que apenas pode ser ocupada por segmentos [+contínuo], bem como a sua velarização nessa posição, que alterna com a glide $[\mathrm{w}]$ no português do Brasil.

Saliente-se que Mateus e Andrade (2000) prescindem do traço [aproximante], presente na proposta seguida pelos autores (Clements \& Hume, 1995), para distinguir as consoantes líquidas das restantes soantes, pelo facto de o traço [contínuo] permitir fazer essa distinção: as nasais caracterizam-se por [+ soante], [- contínuo], enquanto as líquidas são [+soante], [+contínuo].

A distribuição das duas laterais do português (/l/ e $/ K /$ ) é quase complementar, contrastando fonologicamente apenas em posição intervocálica. Com efeito, $/ K /$ está restrito à posição de Ataque no interior de palavra, enquanto /l/ pode ocorrer em Ataque inicial ou medial, sendo também uma das duas consoantes líquidas, a par do rótico / $/ \mathrm{r}$, que pode ocorrer como segundo elemento de um Ataque ramificado e em posição de Coda. O Quadro 2 apresenta a distribuição das laterais em português. 


\begin{tabular}{|l|l|c|}
\hline Constituinte silábico & \multicolumn{1}{|c|}{ /l/ } & \multicolumn{1}{|c|}{$/$} \\
\hline Ataque não ramificado inicial & lata ['late] & - \\
\hline Ataque não ramificado medial & fala ['fale] & falha ['faKe] \\
\hline Ataque ramificado inicial & flauta ['flawte] & - \\
\hline Ataque ramificado medial & atlas ['atlef] & - \\
\hline Coda & $\begin{array}{l}\text { caldo ['kałdu] } \\
\text { sal ['sal] }\end{array}$ & - \\
\hline
\end{tabular}

Quadro 2: Distribuição das laterais em português

Durante o processo de aquisição do português europeu, as laterais em ataque não ramificado são adquiridas tardiamente, decorrendo um longo período de tempo entre a aquisição do primeiro (/l/) e último segmento $(/ K /)$ (Freitas, 2001; Costa, 2010; Amorim, 2014; e.o.). Até se encontrarem estabilizadas, estas consoantes são frequentemente elididas ou substituídas por outra soante, geralmente uma glide. Com menos frequência, encontram-se também substituições por oclusiva (Costa, 2010; Amorim, 2014; Ramalho, 2017).

\section{Metodologia}

Neste trabalho, são analisadas as produções das consoantes laterais em ataque não ramificado, por parte de 80 crianças de ambos os sexos com desenvolvimento típico e falantes nativas dos dialetos setentrionais do PEC. As idades dos informantes variam entre os 3;0 e os 4;11 anos.

Os dados foram recolhidos com base num instrumento original (Amorim, 2014) que consiste numa sequência de cinco ilustrações que formam uma narrativa, permitindo a nomeação das palavras-alvo em fala encadeada. As crianças foram gravadas numa sessão única, tendo sido usado um gravador digital Sony Minidisc MZ-NH900 com microfone unidirecional Lifetech modelo LF 65. Posteriormente, os dados foram transferidos para um computador portátil e foram transcritas foneticamente. Todas as palavras que suscitaram dúvidas foram transcritas por um revisor experiente, tendo-se eliminado da análise todas as que foram transcritas de forma diferente. Também foram eliminadas da análise as produções não coincidentes com o alvo motivadas por assimilação e as que foram sujeitas a epêntese, uma vez que essas estratégias de reconstrução não são motivadas pela estrutura interna do segmento, foco do nosso trabalho.

No quadro 3, apresentam-se os estímulos utilizados para as laterais.

\begin{tabular}{|l|l|l|}
\hline Posição na palavra & \multicolumn{1}{|c|}{ /l/ } & \multicolumn{1}{|c|}{ /א/ } \\
\hline CV inicial & lagarto, lágrima, lápis, lavar, livro, lobo & --- \\
\hline CV medial & $\begin{array}{l}\text { bola, bolacha, cabelo, camisola, castelo, cavalo, } \\
\text { estrela, janelas, relógio, vela, zoológico }\end{array}$ & $\begin{array}{l}\text { colher, joelho, orelha, palhaço, } \\
\text { vermelho }\end{array}$ \\
\hline
\end{tabular}

Quadro 3: Estímulos utilizados para as laterais

Os resultados obtidos, num total de 1508 tokens para /1/ em ataque não ramificado e 516 para / $/$, foram analisados quantitativamente com recurso ao software Golsvarb X (Sankoff, Tagliamonte \& Smith, 2005). Foi realizada apenas a análise unidimensional, que indica a frequência de produção de acordo com o alvo das consoantes em estudo.

Foram utilizados os seguintes critérios para considerar um segmento adquirido:

- produção correta acima de $80 \%$ : segmento adquirido;

- produção correta acima de 50\% (50\%-79\%): segmento em processo de aquisição;

- produção correta inferior a 50\%: o segmento ainda não se encontra adquirido. 
Foram também analisadas as produções não coincidentes com o alvo, de forma a aceder às representações fonológicas das crianças e, assim, contribuir para a caracterização interna das laterais do PEC.

\section{Resultados e discussão}

$\mathrm{Na}$ classe das soantes, as consoantes nasais são as primeiras a ser adquiridas, encontrando-se já estabilizadas na faixa etária 3;0-3;5 anos (Mendes et al., 2009/2013; Guimarães et al., 2014; Amorim, 2014). As consoantes soantes adquiridas em seguida são a lateral /l/ e o rótico dorsal em posição inicial de palavra, na faixa etária 3;0-3;5 anos ${ }^{1}$. No entanto, regista-se uma regressão na aquisição da lateral em ambas as posições na palavra na faixa etária seguinte $(3 ; 6-3 ; 11)$, como ilustra o gráfico que se segue.

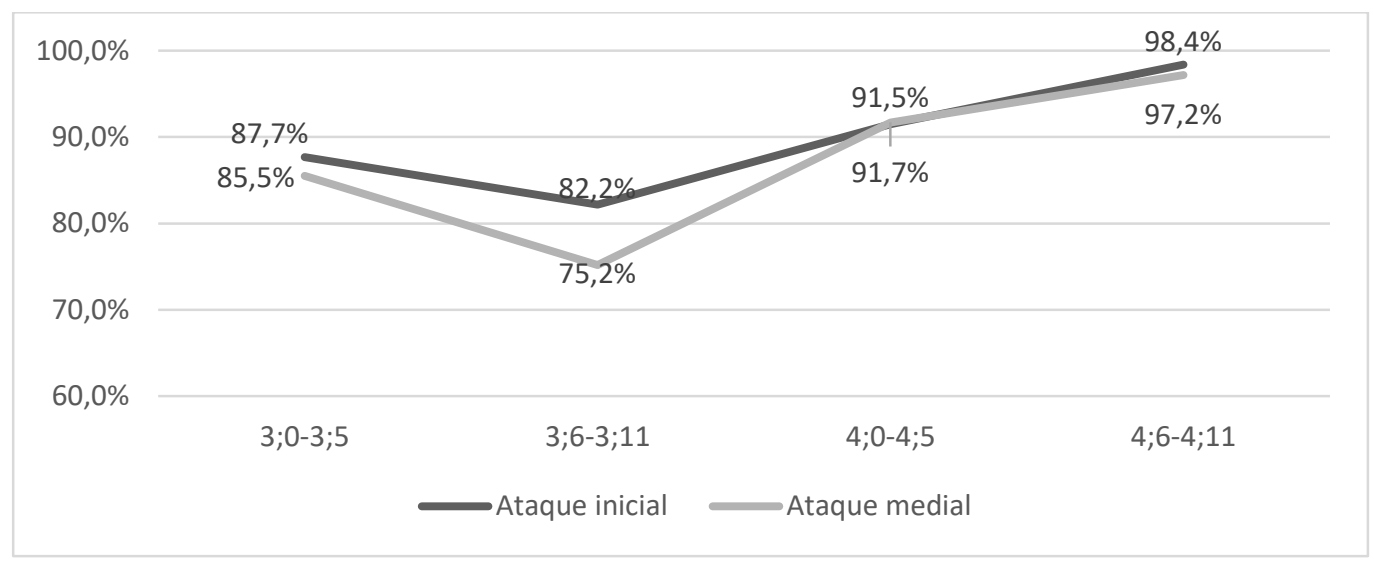

Gráfico 1: Índice de produção de acordo com o alvo da lateral [1]

Esta regressão é mais visível em sílaba interna, posição em que não se ultrapassa a taxa de 73,2\% de produção de acordo com o alvo (Amorim, 2014), pelo que a estabilização efetiva em sílaba interna se dá apenas na faixa etária 4;0-4;05. A regressão registada poderá estar relacionada com a aquisição de ambos os róticos em posição medial, já que a estabilização de /R/ medial e de /r/ se dá na faixa etária 3;6-3;11.

Já a lateral palatal regista um índice de produção de acordo com o alvo acima dos $80 \%$ apenas na faixa etária 4;6-4;11.

Observados os padrões de substituição, verifica-se que a lateral [+ant] é substituída preferencialmente pela glide [w] (49 em 88 das substituições - 56\%) ou pela oclusiva [g] (32\%). Note-se que a maior parte das substituições (76\%) ocorre em sílaba interna. Já a lateral palatal é exclusivamente substituída por outra soante, privilegiando-se a glide [j] (82\% das substituições), a que se segue a lateral [1], que ocorre em $12 \%$ das substituições.

(2) Exemplos de não produção das laterais de acordo com o alvo:

$[1]>[\mathrm{w}, \mathrm{g}]$

$\begin{array}{llll}\text { lápis } & \text { ['lapif] } & \rightarrow & \text { ['wapif] (Rita, 4;10.16), ['gapif] (Lara 3;9.26) } \\ \text { janelas } & \text { [3e'nelef] } & \rightarrow & \text { [3e'newef] (Bernardo, 4;8:11) }\end{array}$

\footnotetext{
${ }^{1}$ Assumimos que, pelo menos nesta etapa de aquisição, o rótico dorsal é processado como uma fricativa (cf. Amorim, 2014; Amorim \& Veloso, 2018), pelo que a primeira soante não nasal a ser adquirida é a lateral alveolar.
} 


\begin{tabular}{|c|c|c|c|}
\hline bolachas & [bu'la $\left.\int e f\right]$ & $\rightarrow$ & [bu'yafef] (Tiago 4;2.11) \\
\hline joelho & [зu' $\mathrm{e} \mathbf{R u}]$ & $\rightarrow$ & [зи' вju] (Beatriz, 3;8.10) \\
\hline colher & [ku' $\mathbf{k} \varepsilon \mathrm{c}]$ & $\rightarrow$ & {$[\mathrm{ku}$ 'jer] (Francisco, $4 ; 4: 22)$} \\
\hline orelha & [ku' $\mathbf{k} \varepsilon]$ & $\rightarrow$ & {$\left[\mathrm{ku}^{\prime} \mathrm{l} \varepsilon\right]$ (Ana Rita $3 ; 1.25$ ) } \\
\hline palhaço & [pe' $\mathbf{K}$ asu] & $\rightarrow$ & [pe'lasu] (João 3;8.4) \\
\hline
\end{tabular}

Note-se também que quando o rótico coronal não é produzido de acordo com o alvo é preferencialmente substituído por [d] (42\% das substituições) ou pela lateral [1] (39\%).

\begin{tabular}{|c|c|c|c|}
\hline pinheiro & [pi'nejru] & $\rightarrow$ & [pi'nejdu] (Manuel 4;6.20) \\
\hline chorar & [ [u'rar] & & [fu'lar] (Rodigo 3;9.24) \\
\hline cadeira & [kg'..pjre[ & $\rightarrow$ & [ke'.rjle] (Afonso 3;10.7) \\
\hline
\end{tabular}

Sendo as representações fonológicas construídas com a aquisição gradual de traços marcados e a sua combinação com traços já adquiridos (Clements, 2005/2009; Fikkert, 2007; Altvater-Mackensen \& Fikkert, 2010; e.o.), a caracterização das laterais como [+lateral, +contínuo] não explica o padrão de substituição encontrado para o rótico alveolar ([r] >> [1]) nem a aquisição da lateral antes do rótico, uma vez que ambos implicariam a aquisição do traço marcado ([+lateral]) antes do não marcado ([-lateral]). Desta forma, colocamse as seguintes questões: i) que traço permite o estabelecimento do contraste entre nasais e laterais no âmbito das soantes? e ii) que traço permite a aquisição do contraste entre laterais e róticos?

Uma vez que o primeiro contraste adquirido no âmbito das consoantes é entre obstruintes e soantes, mais especificamente entre oclusivas e nasais, o traço [+nasal] integra a gramática da criança desde cedo. Por esse motivo, não pode ser este o traço responsável pelo contraste entre nasais e líquidas, no âmbito das soantes, já que isso implicaria que o estabelecimento de um novo contraste fosse resultado da aquisição do traço menos marcado [-nasal].

Considerando a proposta de Mateus e Andrade (2000), o traço [+contínuo] atribuído às laterais permitiria explicar a emergência do contraste entre laterais e nasais: este traço, já presente no sistema da criança no âmbito das obstruintes, estabeleceria uma nova coocorrência com [+soante]. No entanto, se a emergência do contraste entre nasais e líquidas se deve à combinação dos traços [+soante +contínuo], porque emerge a lateral coronal [+anterior] antes do rótico coronal? Tal como referido relativamente ao contraste entre nasais e líquidas, a aquisição do rótico coronal não pode dever-se à aquisição de um traço menos marcado ([-lateral]). Assim, assumimos que, pelo menos nas primeiras etapas de aquisição, as laterais são caracterizadas pelo traço [contínuo].

Tendo sido descartadas as hipóteses de o contraste entre nasais e laterais se estabelecer através dos traços [ \pm nasal], [ \pm contínuo] e [ $[$ lateral], propomos, com base em Lazzarotto-Volcão (2009), que é o traço de raiz [ \pm aproximante] que estabelece o contraste entre soantes nasais e líquidas. Este traço é considerado pouco robusto em Clements (2009), sendo incluído no conjunto "Outros", que ocupa a base da Escala de Robustez. Desta forma, depois de estabelecido o contraste entre obstruintes e soantes através do traço [ \pm soante]), o contraste entre líquidas e nasais, na classe das soantes, seria estabelecido pela aquisição do traço [+ aproximante], que permitiria a emergência do segmento menos marcado, ou seja [+aproximante, -contínuo] $(/ 1 /)$.

A emergência do rótico coronal, que ocorre mais tarde, dever-se-ia à combinação de dois traços marcados já adquiridos: [+aproximante, +contínuo]. Neste caso, o contraste entre laterais e róticos seria estabelecido por [ \pm contínuo]. 
A análise das estratégias de reconstrução utilizadas na não produção do rótico coronal de acordo com o alvo, substituição pelas consoantes [d] ou [1], parece constituir um argumento em favor desta hipótese, já que é explicado pelo apagamento de traços marcados: na substituição pela oclusiva coronal, são apagados os traços [+soante] e [+contínuo]; na substituição pela lateral, é apagado o traço marcado [+contínuo] na combinação com [+aproximante].

Também o padrão de substituições encontrado para a lateral coronal anterior parece reforçar esta hipótese: na substituição pela glide [w], são preservados os traços [+soante, +aproximante], apagando-se o traço [+consonântico]; na substituição pela oclusiva dorsal vozeada [g], preservam-se os traços [-contínuo, +vozeado], mas é usado o traço não marcado [-soante].

\section{Considerações finais}

No estudo apresentado, demonstrou-se que a caracterização das consoantes laterais como [+soante, + contínuo, + lateral], tal como proposto em Mateus e Andrade (2000), não permite explicar a ordem de aquisição das consoantes soantes no português europeu contemporâneo: nasais >> lateral coronal anterior >> rótico coronal. Com efeito, e assumindo que a aquisição segmental é feita pela aquisição gradual de traços marcados e pela sua combinação com traços já adquiridos (entre outros, Costa 2010, Amorim 2015), se as laterais se distinguem dos róticos pelo traço marcado [+lateral], esperar-se-ia que a classe dos róticos fosse adquirida antes das laterais. O facto de /1/ ser adquirido antes dos róticos sugere que o traço [+lateral] não é o responsável por estabelecer o contraste entre as duas classes. Com efeito, considerar que as consoantes laterais são caracterizadas pelo traço [+lateral] implicaria assumir que a emergência de um novo contraste se estabeleceria pela aquisição de um traço não marcado, depois de adquirido o traço marcado.

Tendo em consideração este percurso de aquisição e as características articulatórias das laterais do português, concluímos que o recurso aos traços [aproximante] e [contínuo] permite explicar de forma mais satisfatória a ordem de aquisição encontrada na classe das soantes: primeiro, emergem as consoantes caracterizadas pelo valor negativo destes traços - as nasais, [+soante, - aproximante, - contínuo]) - e só depois as líquidas, caracterizadas pelos traços [+soante, +aproximante]. Por outro lado, a coocorrência de [+aproximante] com [ \pm contínuo] explica também por que emerge primeiro a lateral coronal anterior: assumindo que /1/ é um segmento [-contínuo], a coocorrência do traço [+aproximante] estabelece-se em primeiro lugar com o traço não marcado e só mais tarde coocorre com o traço marcado [+contínuo], o que permite a emergência do contraste entre a lateral coronal anterior e o rótico coronal.

A adoção, na descrição do português, de um traço como [ \pm aproximante] rompe, efetivamente, com as tendências mais estabelecidas no estudo fonológico da língua. Contudo, esta proposta merece, no nosso entender, uma reavaliação mais assertiva. No nosso entender, a adoção de tal traço como um traço pertinente na organização interna das consoantes soantes - nomeadamente distinguindo as laterais dos róticos e das nasais - resolve um problema que os dados de aquisição apresentados e discutidos neste trabalho põem em evidência: perante os dados e a sua discussão nos termos acima desenvolvidos, o traço [ \pm contínuo] não explica a ordem de estabilização das laterais no processo aquisitivo.

Para além da inadequação explicativa que acabamos de referir, convém ainda sublinhar, nestas considerações finais, algumas reservas importantes quanto à aceitação de [ \pm contínuo] como um traço verdadeiramente pertinente na organização fonológica do português:

- na própria identificação de correlatos minimamente estáveis deste traço, confundem-se, conforme foi referido, quer critérios de natureza fonética relacionados com a ambivalência articulatória das laterais que reúnem simultaneamente propriedades dos segmentos [+contínuo] e dos segmentos [-contínuo], quer critérios 
de natureza fonológica relacionados com o comportamento muito variável, do ponto de vista diacrónico e sincrónico, de segmentos como novamente as laterais relativamente à continuidade;

- na subclasse das soantes, [ \pm contínuo] não parece desempenhar verdadeiramente qualquer função distintiva na individualização das laterais, dos róticos e das nasais: no caso das nasais, p. ex., [+nasal], em PEC, implica sempre [-contínuo], tornando este último redundante - e, como tal, sem cabimento na representação subespecificada destes segmentos; no caso das laterais, [+lateral] dispensa também da subespecificação das laterais qualquer informação lexical acerca da continuidade;

- finalmente, refira-se que, em estudos fonéticos anteriores relativos ao português e ao espanhol, na classe das obstruinte, [ \pm aproximante] (e não [ \pm contínuo]) é apresentado também como o traço pertinente na distinção entre oclusivas e fricativas: o processamento das "oclusivas fricatizadas" (foneticamente contínuas) nestas duas línguas como oclusivas e não como fricativas, p. ex., levou propostas anteriores (Martínez Celdrán 1984, 1991; Veloso 1997) a identificar [aproximante] como o verdadeiro traço responsável pela preservação da oposição entre as duas subclasses obstruintes destas duas línguas iberorromânicas.

No seu conjunto, todos estes dados reforçam a pertinência de uma reavaliação da funcionalidade fonológica de [ \pm contínuo], provavelmente um traço eminentemente fonético em português, sujeito a variação e fonologicamente redundante, ou seja, não cabível nas representações lexicais do consoantes do PEC.

\section{Referências}

Altvater-Mackensen, Nicole \& Paula Fikkert (2010) The acquisition of the stop-fricative contrast in perception and production. Lingua 120, pp. 1898-1909.

Amorim, Clara (2014) Padrão de aquisição de contrastes do PE: a interação entre traços, segmentos e sílabas. Tese de doutoramento, Universidade do Porto: FLUP.

Andrade, Ernesto d' (1977) Aspects de la phonologie (générative) du Portugais. Lisboa: INIC, Centro de Linguística da Universidade de Lisboa.

Andrade, Ernesto d' (1998) Variação fonética do /1/ em ataque silábico em Português Europeu. Actas do XIII Encontro Nacional da APL. Lisboa: Colibri/APL, pp. 55-76.

Brown, Cynthia \& John Mathews (1997) The role of feature geometry on the development of phonemic contrasts. In. S. J. Hannahs \& Martha Young-Scholten (eds.) Focus on phonological acquisition. Amsterdam/Philadelphia: John Benjamins, pp. 67-112.

Chomsky, Noam (1986) Knowledge of language: its nature, origin and use. New York: Praeger.

Chomsky, Noam \& Morris Halle (1968) The sound pattern of English. New York: Harper and Row.

Clements, George Nick (2001) Representational economy in constraint-based phonology. In. T. Alan Hall (ed.) Distinctive feature theory. Berlin: Mouton de Gruyter, pp. 71-146.

Clements, George Nick (2009) The role of features in speech sound inventories. In. Eric Raimy \& Charles Cairns (eds.) Contemporary views on architecture and representations in phonological theory. Cambridge, MA: MIT Press.

Clements, George Nick \& Elizabeth Hume (1995) Internal organization of speech sounds. In. John Goldsmith (ed.) The Handbook of Phonological Theory. Oxford: Basil Blackwell, pp. 245-306.

Costa, Teresa (2010) The Acquisition of the Consonantal System in European Portuguese: Focus on Place and Manner Features. Dissertação de Doutoramento, Faculdade de Letras da Universidade de Lisboa.

Dresher, B. Elan (2009) The contrastive hierarchy in phonology. Cambridge: Cambridge University Press.

Dresher, B. Elan, Glyne L. Piggott \& Keren Rice (1994) Contrast in phonology: Overview. Toronto Working Papers in Linguistics 13 (1), pp. iii-xvii. 
Emiliano, António (2009) Fonética do português europeu: descrição e transcrição. Guimarães: Guimarães Universitária.

Fikkert, Paula (1994) On the acquisition of prosodic structure. Dordrecht: HIL.

Fikkert, Paula (2007) Acquiring phonology. In Paul de Lacy (ed.) Handbook of phonological theory. Cambridge, MA: Cambridge University Press, pp. 537-554.

Fikkert, Paul (2010) Developing representations and the emergence of phonology: evidence from perception and production. In. Cécile Fougeron, Barbara Kühnert, Mariapaola d' Imperio \& Nathalie Vallée (Eds.) Laboratory Phonology 10. Berlin / New York: De Gruyter, pp. 227-258.

Fikkert, Paula \& Clara Levelt (2008) How does place fall into place? The lexicon and emergent constraints in children's developing grammars. In. Peter Avery, B. Elan Dresher \& Keren Rice (eds.) Contrast in Phonology: Theory, Perception, Acquisition. Berlin: Mouton de Gruyter, pp. 231-268.

Fikkert, Paula \& Maria João Freitas (2006) Allophony and allomorphy cue phonological development: evidence from the European Portuguese vowel system. Journal of Catalan Linguistics 5, pp. 83-108.

Freitas, Maria João (2001b) Os pontos nos seus lugares: as líquidas na aquisição do português europeu. In. Ivo Castro \& Inês Duarte (eds.) Razão e emoção. Estudos para Maria Helena Mateus. Lisboa: INCM, pp. 307326.

Ingram, David (1989) First language acquisition: Method, description and explanation. Cambridge: Cambridge University Press.

Jakobson, Roman (1941/1968 Child Language, Aphasia and Phonological Universals. The Hague \& Paris: Mouton.

Jakobson, Roman, Gunnar Fant \& Morris Halle (1952) Preliminaries to speech analysis: the distinctive features and their correlates. Cambridge: MIT Press.

Lazzarotto-Volcão, Cristiane (2009) Modelo Padrão de Aquisição de Contrastes: Uma proposta de avaliação e classificação dos Desvios Fonológicos. Tese de doutoramento, Universidade Católica de Pelotas.

Mateus, Maria Helena Mira \& Ernesto d'Andrade (2000) The Phonology of Portuguese. Oxford: Oxford University Press.

Martínez Celdrán, Eugenio (1984) Cantidad e intensidade en los sonidos obstruyentes del castellano: hacia una caracterización acústica de los sonidos aproximantes. Estudios de Fonética Experimental I, pp. 73-129.

Martínez Celdrán, Eugenio (1991) Sobre la naturaleza fonética de los alófonos de /b d g/ en español y sus diversas denominaciones. Verba 18, pp. 235-253.

Mielke, Jeff (2005) Ambivalence and ambiguity in laterals and nasals. Phonology 22 (2), pp. 169-203.

Oliveira, Catarina, Paula Martins, António Teixeira, Isabelle Marques \& Pedro Sá-Couto (2011) An Articulatory and Acoustic Study of the European Portuguese /1/. 17th International Congress of Phonetic Sciences - ICPhS XVII, Hong-Kong.

Peperkamp, Sharon \& Emmanuel Dupoux (2002) Coping with phonological variation in early lexical acquisition. In. Ingeborg Lasser (Org.) The Process of Language Acquisition. Berlin: Peter Lang Verlag.

Ramalho, Ana Margarida Monteiro Cortes (2017) Aquisição fonológica na criança: tradução e adaptação de um instrumento de avaliação interlinguístico para o português europeu. Tese de doutoramento, Universidade Évora.

Veloso, João (1997) As diferentes denominações das "Oclusivas Fricatizadas" do português. Implicações linguísticas da questão. In. Ana Maria Brito et al. (Orgs.) Sentido que a Vida faz. Estudos para Óscar Lopes. Porto: Campo das Letras, pp. 845-854. 